\title{
Platform Variability in Edge-Cloud Vision Systems
}

\author{
Ali J. Ben Ali \\ University at Buffalo \\ alijmabe@buffalo.edu
}

\author{
Sofiya Semenova \\ University at Buffalo \\ sofiyase@buffalo.edu
}

\author{
Karthik Dantu \\ University at Buffalo \\ kdantu@buffalo.edu
}
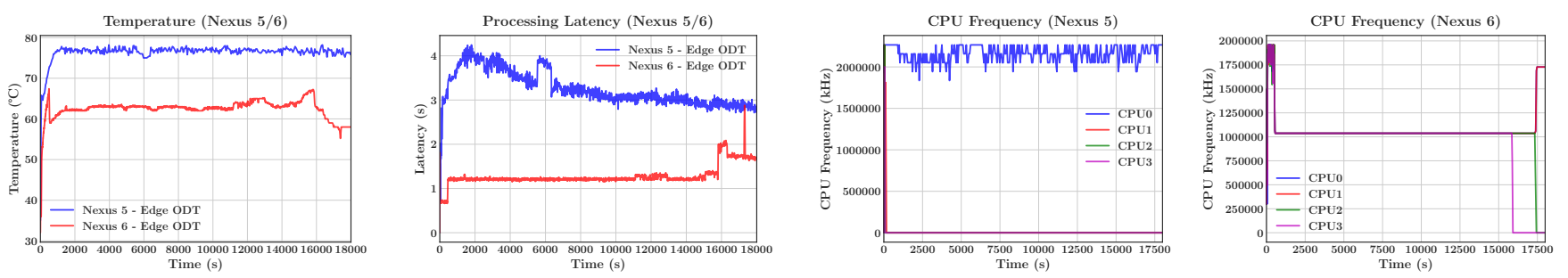

Figure 1: (1) CPU temperature, (2) per-frame processing latency, and (3-4) CPU core frequency for Nexus 5/6

\section{ABSTRACT}

Modern edge devices such as smartphones, tablets, smart glasses, VR and AR headsets have gotten more capable with high-resolution displays, powerful CPU/GPUs, multiple sensing modalities and multiple connectivity options. These platform capabilities are enabling complex sensing applications that integrate visual, inertial, and other sensing modalities in real-time to enable applications such as indoor navigation, object recognition, face recognition, activity recognition, and others. Typically, such applications are computationallyintensive and require execution of complex computer vision pipelines for effective execution.

While edge devices are getting more powerful, it is still challenging to run such computationally intense applications exclusively on the edge. To address this challenge, several solutions propose splitting the computing between the cloud and the edge. However, these solutions are typically tested for a few seconds/minutes. Our observation is that this ignores platform constraints when multiple apps are running on the same edge device and/or long-term operation, resulting in platform variability such as voltage/frequency scaling, shutting down of CPU cores, etc. all of which affect the application performance.

To demonstrate this concern, we perform the following experiment. We build an example edge-cloud vision system - performing Object Detection and Tracking (ODT) using

Permission to make digital or hard copies of part or all of this work for personal or classroom use is granted without fee provided that copies are not made or distributed for profit or commercial advantage and that copies bear this notice and the full citation on the first page. Copyrights for thirdparty components of this work must be honored. For all other uses, contact the owner/author(s).

HotMobile '19, February 27-28, 2019, Santa Cruz, CA, USA

(c) 2019 Copyright held by the owner/author(s).

ACM ISBN 978-1-4503-6273-3/19/02.

https://doi.org/10.1145/3301293.3309555
TensorFlow using the SSD-MobileNet-v2 model. We run the application on two devices - Google Nexus 5 (Qualcomm Snapdragon $8002.26 \mathrm{GHz}$ quad-core processor with $2 \mathrm{~GB}$ memory) and Google Nexus 6 (Qualcomm Snapdragon 805 $2.7 \mathrm{GHz}$ quad-core processor with $3 \mathrm{~GB}$ memory). Figure 1 shows the CPU temperature, processing latency per image frame, and CPU frequency over five hours run. Due to the processing load, the CPU temperature goes above an acceptable threshold at time $=30$ s (Nexus 5) and time $=250$ s (Nexus 6 ). These temperature variations cause the OS to reduce the $\mathrm{CPU}$ frequency in both cases, increasing the processing latency per frame. Further, on the Nexus 6, we observe that the OS turns off some cores to reduce platform temperature after running for more than four hours, while Nexus 5 only runs on one core for most of the time.

These observations have been made while running one application only. As is typical, if multiple applications were running on these edge devices, their performance would be affected further due to the platform load. We also have results from running multiple applications, as well as running the ODT application in a split manner between the edge and fog/cloud for improved performance. In each of these cases, we observe a similar behavior. Our proposal is that future edge-cloud systems need to explicitly test for platform variation due to long-term operation in order to demonstrate feasibility.

\section{ACM Reference Format:}

Ali J. Ben Ali, Sofiya Semenova, and Karthik Dantu. 2019. Platform Variability in Edge-Cloud Vision Systems. In The 20th International Workshop on Mobile Computing Systems and Applications (HotMobile '19), February 27-28, 2019, Santa Cruz, CA, USA. ACM, New York, NY, USA, 1 page. https://doi.org/10.1145/3301293.3309555 